\title{
L'INVENZIONE DELLA SIRENA: MITI MARINI E FIGURAZIONI ALATE NELLA STORIA IMMAGINARIA DEL MEDITERRANEO
}

\author{
THE INVENTION OF THE MERMAID: \\ SEA MITHS AND WINGED FIGURES IN THE IMAGINARY \\ HISTORY OF THE MEDITERRANEAN
}

\author{
Biagio D'Angelo \\ Universidade de Brasília/CNPq, Distrito Federal, Brasília, Brasil \\ biagiodangelo@gmail.com
}

Riassunto: La sirena è uno dei miti mediterranei più proficui tanto in letteratura come nelle arti. Macro-categoria culturale che "spiegava" un'esperienza del reale, il mito della sirena si è talmente trasformato durante i secoli e le culture da parodiare o mantenere appena tenuemente il legame con il celebre episodio omerico. Magritte, ne "L'invention collective", raffigurando una creatura che avrebbe potuto far parte della zoologia fantastica di Borges e Guerrero, presenta all'osservatore, una sirena "al rovescio". Significativamente fuori dall'acqua, la sirena ha gambe umane, femminili e il resto del corpo di un pesce. Già Diderot, nei suoi "Pensées détachées", aveva riferito dell'orrore provocato da una sirena rovesciata. Le sirene, ad eccezione della nomenclatura di alcuni mammiferi marini, non esistono. Tuttavia, come suggerisce Agamben parlando delle "Ninfe", esse sono « reali », poiché rappresentano, come si evince dal titolo del quadro di Magritte, un' "invenzione collettiva », cioè un concetto costruito per dimostrare l'origine di aspetti misteriosi della realtà. L'invenzione "collettiva" del mito della sirena, come Diderot e Magritte avevano osservato, rappresenta un rovesciamento della narrazione mitologica. Nella prospettiva inaugurata da Diderot e Magritte, e attraverso esempi tratti da opere di Giraudoux, Tanizaki e D'Arrigo, intuiamo che la modernità del mito della sirena risieda nell'ambiguità e dualità della sua figura a metà.

Parole chiave: Sirena; mito; immaginario; figura rovesciata; comparazione artistico-letteraria

Abstract: The mermaid is one of the most famous Mediterranean myths, both in literature and in the arts. As a cultural macro-category that "explained" an experience of reality, the myth of the mermaid has been so transformed over the centuries and cultures that it has barely maintained the link with the celebrated Homeric episode. In "The Invention Collective", depicting a creature that could have been part of the fantastic zoology of Borges and Guerrero, René Magritte represents an "upside-down" mermaid. Significantly out of the water, the mermaid has human, female legs and the rest of the body of a fish. Already Diderot, in his "Pensées détachées", had already reported on the horror caused by an inverted mermaid. With the exception of the nomenclature of some sea mammals, mermaids do not exist. However, as Agamben suggests, speaking of the "Nymphs", they are "real", since they represent, as can be seen from the title of the painting by Magritte, a "collective invention", that is, a concept constructed to demonstrate the origin of mysterious aspects of reality. The "collective"

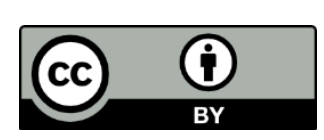


invention of the mermaid's myth, as Diderot and Magritte had observed, represents a reversal of the mythological narrative. In the perspective inaugurated by Diderot and Magritte, and through examples taken from works by Giraudoux, Tanizaki and D'Arrigo, we would like to argue that the modernity of the mermaid's myth resides in the ambiguity and duality of its half figure.

Key-wordls: Mermaids; Myth; Imaginary; Upside-down Figure; Literary-Visual Comparaison

"A lo largo del tiempo, las sirenas cambian de forma" (Borges y Guerrero, Manual de zoología fantástica)

"Écrire, c'est entendre la voix perdue" (Pascal Quignard, Le nom sur le bout de la langue)

\section{Voci e silenzi}

Scrivere sulle sirene è, come a giusta ragione propone Pascal Quignard, citato in esergo, ascoltare una voce perduta, rimettersi all'ascolto di storie e miti, le cui variazioni non cessano di interrogare le letterature e le arti plastiche. La sirena, com'è noto, è uno dei miti mediterranei più proficui tanto in letteratura come nelle arti. Macro-categoria culturale che spiegava un'esperienza del reale, il mito della sirena si è talmente trasformato durante i secoli e le culture da parodiare o mantenere appena tenuemente il legame con il celebre episodio omerico. Creatura degli oceani e dei mari, ma anche dell'aria e delle rocce, semi-pesce, semidonna, semi-ninfa, semi-uccello, la sirena, riunendo in sé il grido disperato e il canto armonioso, appartiene a due mondi separati, quello dei cieli e degli inferi, il mondo dei vivi e dei morti. La forza del canto delle sirene consiste nella loro ambiguità rivelatrice e ingannevole, ammaliatrice e mortifera al tempo stesso, un'energia comunicatrice enigmatica e meravigliosa. Le sirene, direbbe Foucault, "non sono che canto", "promessa ingannevole e verace al tempo stesso": "Le sirene sono la forma inafferrabile e proibita della voce che attira, esse non sono che canto [...] Offerto come risucchio, il canto non è che l'attrazione del canto, ma non promette nient'altro all'eroe che il doppio di ciò che egli ha già vissuto, conosciuto, sofferto: nient'altro che ciò che egli è" (Foucault 125-126).

Nella riproposizione del mito, si riscontra un denominatore comune: il rapporto tra la voce delle sirene e la scrittura emerge come un vero e proprio scontro tra materialità. Non a caso Quignard rileva che il linguaggio - ogni linguaggio - manca inevitabilmente di un quid. E a sua volta, la voce - la voce dell'artista e quella delle sirene - persegue, nostalgicamente, un luogo che possa recuperare la propria soddisfazione precaria e incompleta. Sappiamo, tuttavia, che la voce delle sirene nasconde pure una minaccia che aveva interrogato Kafka, un'arma ancor più terribile del loro stesso canto: il loro silenzio. Si potrebbe addirittura riuscire a sfuggire al loro canto, ma non certamente al loro silenzio: "Au sentiment de les avoir vaincues par sa propre force et à l'orgueil violent qui en résulte, rien de terrestre ne saurait résister" (Kafka 542). Kafka spinge il mito sirenico alla sponda del terrore del silenzio poetico. Ulisse è scampato al loro canto. E se invece le sirene avessero semplicemente taciuto, l'eroe omerico avrebbe ancora fatto esperienza della salvezza? Per Evanghelia Stead, le sirene kafkiane rappresentano quel fervore verso i miti, dei quali si sottolinea "un penchant pour l'érudition et la glose. Il n'est en ce sens qu'une fable moderne sur la menace que fait peser la science sur la 
séduction du chant" (Stead 316). Il silenzio è un'altra invenzione affascinante del mito delle sirene, un'ulteriore possibile variazione del mito nella geografia omerica mediterranea.

A questo silenzio, però, Barthes, insoddisfatto, avrebbe indicato una sortita suggestiva: “Est-ce que j'entends des voix dans la voix? - Mais n'est-ce pas la vérité de la voix que d'être hallucinée? L'espace de la voix n'est-il pas un espace infini?" (Barthes 240).

Resistere alle sirene sarebbe dunque arduo e impossibile. Se la scrittura è udire la voce perduta, sotto forma di allucinazioni, proprio questo magnetismo è sirenico, irrefrenabile, quasi suicida. Decidere di abbandonarsi al canto delle sirene è castigabile. L'eroe sfiderebbe, altrimenti, le leggi della natura a lui vietate. Quello delle sirene è, difatti, un canto fatato e menzognero:

\begin{abstract}
È un canto che è una promessa: se si fermerà presso di loro, se ne andrà «sapendo più cose». Le sirene, pur consapevoli della loro voce di miele, sanno che è irresistibile, per gli uomini che arrivano a sentirla, non tanto la dolcezza del canto, quanto il conoscere il proprio passato [...] (Esse) mentono o, incoerenza del mito che le vuole onniscienti, non sanno che il desiderio di «sapere più cose» ha portato tutti coloro che si sono fermati presso di loro per soddisfarlo a dimenticare gli affetti familiari, a trascurare tutto ciò che ha a che fare con la vita, fino a lasciarsi morire: sembrano non rendersi conto che, dal mare, si possono vedere tra i fiori, le loro ossa e loro membra imputridite... La bella voce è solo l'involucro della vera tentazione delle sirene omeriche: «sapere più cose». È la tentazione «originaria» dell'onniscienza. Cedere a questa tentazione, assecondare, in modo assoluto, questo desiderio porta a rompere $\mathrm{i}$ legami famigliari, a perdere la dimensione sociale e civile, a morire. Per questo Omero le condanna. Per questo l'eroe deve fuggirle, non deve interrompere il suo nóstos. (Canavero 134)
\end{abstract}

Abbiamo dunque due versioni apparentemente contradittorie di queste figure mitologiche: esse sono leggiadre e gentili, dalla voce straordinaria, disponibili al sacrificio del loro stesso corpo, pur di restare fedeli all'amicizia di Proserpina; esse, però, sono anche causa di morte e di disorientamento fisico e psicologico dei mortali. Il loro canto assume, in questo caso, una funzione crudele, mortifera. Al tempo stesso esso incanta poiché evoca liricamente le grandi questioni ultime del destino umano. "Forse si può avere ragione di immaginare il canto degli angeli e delle sirene come il miracolo sonoro di una biunivocità siffatta, che nulla interviene a offuscare" (Sloterdijk 99). La sirena è un angelo, nuovo Adamo, che potrebbe rivelare all'uomo la natura divina, ma porta in sé una promessa di distruzione.

Cantando o, in altre parole, distillando un dolce veleno nelle orecchie, le sirene "spiegano" cos'è la morte, cos'è il destino. Un eccesso di sapere, che rivela, nientedimeno che il senso recondito dell'esistenza. Creature semidivine, ninfe messaggere della verità, molto prossime agli dei dell'Olimpo, le sirene, con il loro canto, ammaliano perché promettono agli uomini una conoscenza totale dei misteri, tutta la verità. Le sirene sono il serpente biblico, travestito da figure ibride nell'immaginario mitologico greco-antico. Come il serpente, resistere al loro fascino è impossibile e fatalmente mortale.

Tuttavia, ancor più pericolosa, perché più ambigua e seducente, per il desiderio d'orrore che essa provoca, lo è una sirena «rovesciata».

Magritte, ne L'invention collective, raffigurando una creatura che avrebbe potuto far parte della zoologia fantastica di Borges e Guerrero, presenta all'osservatore, una sirena al rovescio. Significativamente fuori dall'acqua, la sirena ha gambe umane, femminili e il resto del corpo di un pesce. Già Diderot, nei suoi Pensées détachées, aveva riferito dell'orrore provocato da una sirena rovesciata. 
Le sirene, ad eccezione della nomenclatura di alcuni mammiferi marini, non esistono. Tuttavia, come suggerisce Agamben parlando delle Ninfe, esse sono «reali», poiché rappresentano, come si evince dal titolo del quadro di Magritte, un "invenzione collettiva", cioè un concetto costruito per dimostrare l'origine di aspetti misteriosi della realtà. L'invenzione collettiva del mito della sirena, come Diderot e Magritte avevano osservato, rappresenta un rovesciamento della narrazione mitologica.

Se seguiamo la prospettiva inaugurata da Diderot e poi, sotto la lente surrealista, da Magritte, la modernità del mito della sirena risiede proprio nell'ambiguità e dualità della sua figura a metà. Mostruosa e, allo stesso tempo, affascinante, la sirena sembra dichiarare che le forme sono fallaci, che le apparenze sono illusorie e che il vero pilastro epistemologico del sapere oggi consiste nell'incertezza del visibile e del narrabile.

Nel celebre saggio sulle Ninfe, Agamben, ripercorrendo il concetto di Pathosformeln di Aby Warburg, ricorda quali emblemi antropologici sono nascosti nella ripresa del mito delle sirene: "L'umano si decide in quella terra di nessuno fra il mito e la ragione, nell'ambigua penombra in cui il vivente accetta di confrontarsi con le immagini inanimate che la memoria storica gli trasmette per restituire loro vita" (Agamben 34-35).

Che cosa sono, allora, le sirene? Incubi, fantasmi, simulacri? Cosa ci dicono certe riletture del mito classico?

\section{Sirene rovesciate}

Indubbiamente, risulta molto produttivo ripensare al concetto warburghiano di Nachleben per il quale la storia delle immagini e dei testi riprenda significato a partire da miti e figure del passato. Il loro significato, però, non rimane immutato, ma si contestualizza ed è sottoposto a nuove metamorfosi nel tempo e nello spazio. Alla sopravvivenza corrisponde, come ricorda Didi-Huberman, un rinnovarsi del senso: "Ciò di cui le sopravvivenze serbano memoria non è il significato - che cambia a ogni movimento e in ogni contesto - in ogni rapporto di forze in cui è incluso - ma lo stesso tratto significante" (Didi-Huberman 169). La descrizione visionaria che ne fa Laura Pugno, in un romanzo del 2007, fa delle sirene albine delle creature mostruose, studiate dagli scienziati in un futuro apocalittico perché immuni al cancro alla pelle:

\footnotetext{
C'era chi credeva che le sirene fossero una mutazione genetica, un'evoluzione dei dugonghi o lamantini quasi estinti, per fronteggiare un mondo da cui l'essere umano era destinato a sparire. Altre creature, suboceaniche, avrebbero dominato la Terra. Altri sostenevano che era normale scoprire specie sconosciute, visto che l'uomo era ormai in grado di abitare il fondo dei mari e degli oceani, anche se ci volevano molti soldi per farlo. Specie nuove, o forse antichissime, come le meravigliose sirene crudeli. (Pugno 16)
}

Prendendo spunto da un'antica tradizione sulle creature sireniche, parodiate già da Luciano di Samosata (vedasi il suo De dea Syria), ci interessano tre sirene moderne "rovesciate" che appartengono a culture differenti (la giapponese, la francese e la siciliana) e che guardano alla mediterraneità come lo spazio discorsivo a cui attingere per ricreare i miti e interrogare la storia.

$* * *$

Nel lontano Giappone, Tanizaki Jun'ichirō pubblica il racconto «Pianto di sirena» (人 魚の嘆き, “Ningyo no nageki”). Siamo nel 1917. È certamente una geografia distante dalla 
storia immaginaria del Mediterraneo. Tuttavia, non è del tutto fuori luogo. Riassumiamo la vicenda narrata. Un'incantevole sirena è acquistata dal nobile Meng Shishou in un imprecisato passato cinese quasi fiabesco. Insoddisfatto con la vita e desiderando la Bellezza più di qualsiasi altra cosa al mondo, il nobile personaggio si lascia convincere, da un mercante olandese che era giunto fino ai mari della Cina, di comprare una straordinaria e bellissima sirena. Conquistato dalla bellezza, ma anche dalla stranezza della figura umano-marina, Meng Shishou, meravigliato, acquista la sirena e passa il tempo alla sua contemplazione malinconica, conscio che non potrà mai possederla appieno. La sirena, infatti, è prigioniera di una gigantesca teca di vetro che la contiene. La sirena, tuttavia, convince il nobile, con seduttorie orazioni, languide e pacate, a liberarla. Una volta ritornata ai mari occidentali, Meng Shishou decide di seguirla, verso un viaggio indefinito, "verso la bramata e adorata Europa, verso il Mediterraneo, luogo natio della sirena" (Tanizaki 88). Chiediamoci, a questo punto, a quale tradizione mitologica Tanizaki si riferisce scrivendo "Pianto di sirena". Infatti, Tanizaki utilizza il termine ningyo, della mitologia nipponica. Tuttavia, siamo di fronte a una ningyo rovesciata.

Secondo Michael Dylan Foster (2014), una ningyo non corrisponde alla sirena del contesto mitologico occidentale. Iconograficamente, la parola ningyo identifica piuttosto una creatura dal torso umano, una bocca di scimmia e i denti di pesce. Uno studio di mitologia comparata sarebbe a questo punto necessario: la brutta sirena nipponica, però, possiede una coda di pesce dalle scaglie dorate e una voce dolce che rassomiglia, come nei miti marini europei, al suono di un flauto o del canto di un uccello. Unico e terribile svantaggio: incontrare o pescare una sirena sarebbe l'avviso di una disgrazia o di una catastrofe. Secondo la leggenda giapponese, se un pescatore dovesse prendere nelle reti una sirena, dovrà immediatamente ridonarla agli oceani. Non riconoscere una ningyo equivale ad attrarre su di sé e sul proprio paese guerre e altre calamità nefaste.

Come proposto dallo stesso racconto, la sirena di Tanizaki appartiene, dunque, più all'immaginario occidentale che a quello giapponese, e soprattutto alla tradizione che ha generato l'immagine delle sirene seduttrici di Ulisse ed Enea. Inoltre, la sirena di Tanizaki piange. Non capiremo mai se il suo pianto è un'altra arma incantatrice o il desiderio sincero di affrancarsi dalla prigionia degli umani. Tanizaki non lo svela appieno, e anche il suo lettore ne rimane perplesso. La sua sirena mediterranea, smarrita tra i mari orientali, supera la dicotomia limitatrice della sirena che riunisce (o separa) la vita e la morte. Ciò che più interessa a Tanizaki è che la sirena trascini con sé non solo la bellezza, ma pure l'enigma di sé stessa.

$* * *$

Nel breve racconto "Les sirènes", Giraudoux reinterpreta in chiave comico-parodica l'episodio delle sirene dell'Odissea: Ulisse non è il solo a sopravvivere alle mortifere figure alate. Elpénore, il più sciocco dei marinai, assiste, inconsapevole, alla tragica morte dei suoi compagni, perché sotto effetto di droghe. Le sirene non l'hanno ammaliato. Sono rimaste figure alate della letteratura e della mitologia, cioè della menzogna.

Non vi è alcuna bellezza nelle sirene di Giraudoux. Le sirene attirano di solito gli uomini fino a farli schiavi e sopraffarli. Sono astute, romanticamente perverse, al punto che i marinai si getterebbero in mare pur di possederle o pur di morire. Cupio dissolvi o l'eterno ritorno ciclico del mito di Eros e Thanatos sotto mentite spoglie? Le sirene non attirano Elpénore. Così si salva. Senza attrazione, senza fascino. È stanco di credere nelle favole dei mostri. Sembra un degno erede della tradizione diderotiana: "C'est au goût de créer des monstres. Je me précipiterais peut-être entre les bras d'une sirène; mais si la partie qui est 
femme était poisson, et celle qui est poisson était femme, je détournerais mes regards" (Diderot 762).

Le sirene darrighiane, invece, svelano le simbologie infinite del Mediterraneo e dei suoi abitanti. Esse abitano il luogo originale del mito, lo stretto di Messina ${ }^{1}$, campo d'azione di quel romanzo-mondo che è Horcynus Orca. Sono figure notturne, che personificano l'amplesso metaforico tra l'esistenza precaria, alla ricerca di un senso, e il sesso e la sensualità, tentativi di risposta ai desideri umani attraverso un'animalità spesso censurata.

Le "femminote", le sirene di cui si serve D'Arrigo come materiale indispensabile della narratività di un mondo mitico allo sfacelo, sono sprovviste di risposte al bisogno di erotismo ancestrale di 'Ndria Cambria, ma consentono di operare una trasformazione nel personaggio. Infatti, non solo il corpo sembra modificarsi, in un eterno fluire di corporalità e sensazioni intime, ma soprattutto l'anima, che comincia a respirare ed esistere in una nuova traslocazione, quasi un nuovo nomadismo interiore che si perpetua ab aeterno, e che manifesta la forza della natura e della vita, al di sopra della morte e della degradazione delle forme e delle sostanze. Donne contrabbandiere, così le raffigura D'Arrigo, in una quasi incosciente "sopravvivenza" warburghiana: "Femminote e fere, nei caratteri, in tutto, si trattavano, le une con le altre, come si meritavano, e forse c'era del vero in quello che sosteneva don Mimi Nastasi, che cioè erano intrinseche e avevano lo stesso sangue, perché discendevano tutte e due, per gradi, dalle sirene" (D'Arrigo 153).

Don Mimì è a suo agio tra le sirene. "Le aveva inventate lui", osa scrivere D'Arrigo: "Fra gli ami don Mimi era nel suo, com'era in mezzo alle sirene" (D'Arrigo 667). "Parlava sempre e solo di sirene... e se fosse, sotto sotto, una sirena maschio, questo don Mimi? si dicevano, se fosse giusto giusto lui il maschio fatto su misura per le sirene? che ne sappiamo noi? e che ne sa lui?" (D'Arrigo 668).

Tra la sirena e la "femminota" si instaura un ponte tra sistemi cosmici: "Ma queste femmine sirene erano come le nostre madri, le nostri sorelle? gli facevano frequentemente per invitarlo $[\ldots]$ Le sirene invece sono un'altra cosa, altra razza di femmine: quelle, fatevi conto, sono femmine femminote" (D'Arrigo 676).

La donna-pesce è per D'Arrigo speranza e sventura, demoniaca e divina, mitica e mistica, incarna la voluttà e la saggezza che si cerca con cupidità. La sirena darrighiana riunisce la natura e la cultura, il mito e la storia, il grido disperato e il bisogno affettivo, il canto e il silenzio. La "femminota" è lontana parente della sirena evocata, non poche volte, da Charles Baudelaire.

Nei Fleurs du Mal, Baudelaire offre un'immagine audace della sirena: "Prends le masque d'une sirène/ Faite de chair et de velours", scrive il poeta. La sirena è qui invocata come

\footnotetext{
${ }^{1}$ Le sirene della mitologia greca sarebbero le figlie del fiume Acheloo e, probabilmente, della musa Calliope. Amiche e compagne fedeli di Proserpina, che è brutalmente rapita da Platone, dio degli Inferi, nel fondo dei mari, le «dotte Sirene», come le denomina Ovidio (2005, libro V, 552-563), chiedono a Giove, padre di Proserpina, e ottengono di essere metamorfizzate in «sirene», appunto, creature ibride, metà donne e metà pesci, per poter restare per sempre in compagnia della sciagurata Proserpina. In origine, le sirene erano metà donne e metà uccelli. Ovidio stesso non fa nessun riferimento alla coda di pesce, ma parla del mare come il luogo in cui le giovani ammaliatrici avrebbero desiderato restare per cercare disperatamente e senza sosta la figlia vergine di Cerere, Proserpina, usurpata e schiavizzata. Queste creature abiterebbero allora all'entrata dello stretto di Messina, in Sicilia. Grazie al loro canto magico e seduttore, ai loro flauti e le loro cetre, le sirene della mitologia greca sono ritenute la causa della perdita del senso dell'orientamento dei naviganti, le cui imbarcazioni si distruggevano sulle rocce e che perdevano la vita sempre a mano di esse.
} 
maschera, travestimento. La sirena di Baudelaire è inafferrabile, incomprensibile, desiderabile, sospesa tra passione e ragione, tra equilibrio e violenza. Angelo o sirena?, si domanda il Poeta nel celebre Hymne à la Beauté :

De Satan ou de Dieu, qu'importe ? Ange ou Sirène?

Qu'importe, si tu rends, - fée aux yeux de velours,

Rythme, parfum, lueur, ô mon unique reine ! -

L'univers moins hideux et les instants moins lourds ? (Baudelaire 25)

Angelo, sirena o fata: la sirena è una bellezza divina e infernale al tempo stesso. La sirena baudelairiana ricongiunge la "belle dame sans merci" di keatsiana reminiscenza e la senhora dei poeti provenzali.

La sirena di Horcynus Orca appartiene esattamente a questi due mondi, il sans merci infernale e la senhora celestiale, i mari e la terra, il mondo dei vivi e il mondo dei morti. Simbolo di un'alterità impenetrabile, la sirena darrighiana è il paradigma allegorico dei pericoli del viaggio esistenziale. O dal mare o dalla rocca, il suo punto d'origine è abissale. E verso questi maelstrom oscuri trascina chiunque si domandi sulla sua genesi.

\section{Riscritture infinite}

Nel tempo, il mito della sirena è stato costantemente reinventato. La sua «invenzione» ha liberato il mito antico dalla sua metafisicità, ma senza diminuire la sua funzione problematica, tragica e concettuale.

Negli esempi che abbiamo offerto, abbiamo assistito a un rovesciamento parodico della figura della sirena. Questa scrittura parodica, accentuata dall'iconografia della sirena «al rovescio», spinge la questione mitologica verso un territorio in cui la realtà diventa, a sua volta, «narrabile». Infatti, la realtà che il mito rivela è sempre fittizia e mitologica. Dal desiderio di conoscenza all'illusione della conoscenza. Nel regno del mito, la finzione è l'unica verità. Essa si unisce a un modulo, per così dire, didattico e esplicativo del reale. Si tratta, però, di un didattismo che vuol essere uno strumento di trasmissione del sapere: spiega, ma non risolve, cerca le ragioni e trova illusioni, trasformando così il mito in un metodo insoddisfacente ma generatore di molteplici storie.

Le sirene hanno il potere di metamorfizzare il soggetto che le ascolta. Immobili sulle rocce o nei fondali marini, le sirene, gorgoni di una bellezza mortifera, provocano l'uomo. La metamorfosi principale consiste nel desiderio, perché l'inquietudine del soggetto è aspetto irresolubile della condizione umana, e anch'essa partecipa del flusso continuo ed enigmatico delle trasformazioni degli esseri, in qualche modo, dell'Essere.

Da Tanizaki a D’Arrigo, passando per Giraudoux, gli autori lasciano dubitare il loro lettore dell'irrealtà stessa dei miti. Le sirene sono là, appollaiate, o nelle acque, pronte a cantare e a rivelare i segreti, o a restare in un tragico silenzio divino. Solo il desiderio muta. Comico, drammatico, erotico, l'uomo si piega al sacrificio: ecco la sua metamorfosi. Il mito è veramente, totalmente antistorico? Quale Bellezza è stata smarrita per sempre? Chi mai potrà rivelarcela? Il dubbio mitico invade le frontiere del sacro. Ma stavolta il sacro non è più un discorso che offre delle risposte prefabbricate. Certo, esso può essere ancora considerato come strumento di comprensione delle dinamiche della realtà, ma non è utilizzato che narrativamente, senz'alcuna pretesa di spiegazione totalizzante. Il mito funziona, allora, come un dispositivo in cui si gioca una dinamica di assenza-presenza. Il patrimonio sacro non è più indispensabile, pur se ancora 
rispettato. A tale assenza di senso del sacro, l'artista risponde con un'altra presenza: l'immagine allegorica della trasformazione dei miti, e non solo della realtà. L'immagine della sirena è dunque il segno di una realtà che svanisce, un miraggio che riflette dei problemi profondi e angosciosi. Per l'artista, si tratta di un'immagine che si mantiene all'interno di una finzione narrativa, che, in un gioco di specchi, svela l'apparenza delle cose, così come l'illusione delle arti.

Il mito si riscrive così in opposizione alla vocazione di certezze che caratterizzava l'hybris dell'homo tecnologicus del XX secolo. L'unica certezza per Tanizaki, D'Arrigo e Giraudoux è l'ambivalenza del soggetto, il suo procedere verso il Nulla e, allo stesso tempo, verso il suo inesauribile desiderio di Bellezza. Le sirene sono reali, direbbe certamente Agamben, ma esse sono apparenza, apparenza reale, e la realtà non è che inganno, uno spettacolo di errori, dove tutto è relativo e instabile, anche i desideri fugaci e i sentimenti contraddittori per definizione.

Il mito si libera, allora, della sua metafisicità, sebbene la sua funzione sacra, tragica e concettuale non ne esca per nulla ridotta. Certo, il mito non è più (e forse non lo è mai stato) un blocco monolitico di domande e risposte. In esso permane, invece, il tentativo di interrogare la realtà vista come sembianza ingannevole delle forme, l'incertezza come pilastro epistemologico, il paradosso dei realismi che le letterature promulgano come loro principale attività nella mimesis.

Diderot e Magritte sanno bene che rovesciare il mito, metamorfizzarlo, è un'operazione che provoca il lettore e lo spettatore. Quest'ultimo, infatti, potrebbe ancora sospettare che nella realtà già trasformata dal mito, possa tuttavia esistere qualche indizio di ciò che era (spirituale o oggettivo). Diderot e Magritte insegnano che la metamorfosi del mito non è mai completa, totale, perché un frammento di sirena potrebbe occultarsi in qualche brandello di realtà nuova. Anche la sirena rovesciata riappare in altra parvenza: beffata dal marinaio ubriaco di Giraudoux, trasformata in "femminota" e progenitrice della Donna in Horcynus Orca, sfuggente e imprendibile nel "Pianto" di Tanizaki.

La sirena è comunque una figura mostruosa, perché essa prefigura la Bellezza, e la Bellezza possiede, nella sua ontologia, qualcosa dell'orrore dell'uomo. Non l'orrore dettato dalla paura, ma da ciò che è talmente Altro-da-sé che fa paura, il Volto inconoscibile, cui si tende per natura.

Invece Giraudoux, in conclusione, sceglie di rompere l'incantesimo delle sirene, spezzando il binomio mitologico di Bellezza e Morte. La scelta della sua rilettura del mito in chiave ironica è una presa di distanza dal mito antico. Il vero significato, per l'autore di Susanna e il Pacifico, consiste nel fatto che il mito sirenico ha bisogno di essere rovesciato per essere compreso. Ma cosa si comprenderà? Nulla, sembra dire Giraudoux. Tutto è un gioco di chiaroscuri, un teatro d'ombre, pieno di impurezza, che solo a chi si allontana dall'oggetto del desiderio è dato percepire. Elpénore è sciocco, malandato, oppiomane e beone. La sua opacità gli permette vincere le sirene. Un elogio dell'opacità, invece che un elogio dell'enigma del mito sirenico?

È un interrogativo che rovescia ancora una volta il mito mediterraneo delle sirene e offre una nuova strada interpretativa, sempre alla ricerca insoddisfatta di una rivelazione. 


\section{Riferimenti}

Agamben, Giorgio. Ninfe. Torino: Bollati Boringhieri, 2007.

Barthes, Roland. L'obvie et l'obtus. Essais critiques III. Paris: Seuil, 1982.

Baudelaire, Charles. Euvres complètes. Bibliothèque de la Pléiade. Préface et notes de Claude Pichois. Paris: Gallimard, 1975.

Borges, Jorge Luis and Margarida Guerrero. Manual de zoología fantástica. Buenos Aires: Fondo de Cultura Económica, 1957.

Canavero, Alessandra Tarabochia. Sirene, un canto per l'anima. In: AA.VV., I Greci. Il sacro e il quotidiano. Milano: Silvana Editoriale, 2004, p. 131-139.

D’Arrigo, Stefano. Horcynus Orca. Milano: Oscar Mondadori, 1982.

Diderot, Denis. Euvres esthétiques. Paris: Garnier, 1959.

Didi-Huberman, Georgers. L'immagine insepolta. Aby Warburg, la memoria dei fantasmi e la storia dell'arte. Torino: Bollati Boringhieri, 2006.

Foster, Michael Dylan. The Book of Yokai. Mysterious Creatures of Japanese Folklore. Berkeley: University of California Press, 2014.

Foucault, Michel. La pensée du dehors [1966]. In italiano, Il pensiero del di fuori. Euridice e le Sirene. In Scritti letterari. Milano, 1984, p. 125-126.

Kafka, Franz. Le silence des Sirènes. In Euvres complètes. T. II. Paris: Gallimard, 1988.

Pugno, Laura. Sirene. Venezia: Marsilio, 2017.

Quignard, Pascal. Le nom sur le bout de la langue. Paris: Gallimard, 1993.

Sloterdijk, Peter. Sfere. Microsferologia. Bolle. Vol. 1. Roma: Meltemi, 2009.

Stead, Evanghelia. Le silence des Sirènes. In Ulisse da Omero a Pascal Quignard, Atti del convegno internazionale, Verona, 25-27 maggio 2000, a cura di Anna Maria Babbi e Francesca Zardini. Verona: Fiorini, 2000, p. 293-317.

Tanizaki, Jun'ichirō. Pianto di sirena e altri racconti. Traduzione di Adriana Boscaro. Milano: Feltrinelli, 2009.

Recebido em: 01 de agosto de 2020 Aceito em: 09 de novembro de 2020 Publicado em dezembro de 2020 